\title{
Solving the Carbon Dioxide Emission Estimation Problem: An Artificial Neural Network Model
}

\author{
Abdel Karim Baareh \\ Computer Science Department, Ajloun College, Al-Balqa Applied University, Ajloun, Jordan. \\ Email: baareh@gmail.com \\ Received May $9^{\text {th }}, 2013$; revised June $10^{\text {th }}, 2013$; accepted June $17^{\text {th }}, 2013$ \\ Copyright (C) 2013 Abdel Karim Baareh. This is an open access article distributed under the Creative Commons Attribution License, \\ which permits unrestricted use, distribution, and reproduction in any medium, provided the original work is properly cited.
}

\begin{abstract}
Climate Pollution due to the Carbon Emission $\left(\mathrm{CO}_{2}\right)$ from the different fossil fuels is considered as a great and important international challenge to many researchers. In this paper we are providing a solution to forecast the poison $\mathrm{CO}_{2}$ gas emerged from energy consumption. Four inputs data were considered the global oil, natural gas, coal, and primary energy consumption to build our system. In this paper, we used the Artificial Neural Network (ANN) as successful and powerful tool in handling a time series modeling problem. The proposed ANN model was used to train and test the yearly $\mathrm{CO}_{2}$ Emission. The data were trained from year 1982 to 2000, and tested for the year 2003 to 2010. From the results obtained we can see that ANN performance was Excellent and proved its efficiency as a useful tool in solving the climate pollution problems.
\end{abstract}

Keywords: Fossil Fuels; Carbon Emission; Forecasting; Artificial Neural Network; Back Propagation

\section{Introduction}

Forecasting the future events is a great, important and risky task that attracted many researchers in different fields. This type of problems contains many variables that should be studied, highlighted, and considered to build the suitable models. The world events and processes should be clearly explained and obviously stated to be processed. Climate pollution due to the carbon emission became an important and serious problem that affects the countries from the different aspects, health, climate, agriculture, economics, and tourism. Adjusting the energy policies is a necessary process to void pollution problem, and keeping the atmosphere clear and clean [1]. All the future reading indicates the increase in $\mathrm{CO}_{2}$ and greenhouse gas emission [2]. Many countries today have commitments between them to reduce the greenhouse gas emission, like the Kyoto protocol and the United Nations (UN) agreement to keep checking the $\mathrm{CO}_{2}$ emission percentage in the atmosphere in order to reduce it to the desired levels [3]. Many scientists consider the global warming due to $\mathrm{CO}_{2}$ emission is dangerous and threat the world more than terrorism. Many countries such as the UK Government's stated a clear objective in order to reduce the $\mathrm{CO}_{2}$ emissions by $10 \%$ from the 1990 base by 2010 and in parallel to generate $10 \%$ of the UK's elec- tricity from renewable sources by 2010 . Renewable electricity has become equal with $\mathrm{CO}_{2}$ reduction [4]. Several studies were developed to find out the relationship between the different energy consumption and $\mathrm{CO}_{2}$ emission $[5,6]$. For all that there is a need to develop a non linear model that estimates the carbon dioxide emission. In this paper we used the artificial neural network as a powerful, capable tool in handling such type of modeling process. ANN was largely used in solving different problems in numerous fields such as Rainfall-runoff, water quality, sedimentation and rainfall forecasting. ANN also proved its efficiency and strength in different number of applications [7,8] such as sales prediction [9], shift failures [10], estimating prices [11] and stock returns [12]. In our case we explored the effect of four inputs variables the global oil, natural gas (NG), coal, and primary energy (PE) consumption on the $\mathrm{CO}_{2}$ emission estimation.

\section{ANN Back Propagation Algorithm}

Artificial Neural Network (ANN) main work is to process the information supplied to the network. It consists of a number of neurons distributed in different layers, these neurons learns by example and trials, the network work according to that and change its weight several times 
reaching to the optimal weights numbers and values, reaching to the desired output from the given desired input. ANNs simulate the human biological nervous systems, and the way it works is similar to the way the brain process information [13]. ANNs proved its strength and efficiency in solving numerous problems in different world fields such as business [14], forecasting [15], feature extraction [16], classifications [17,18] etc. In this paper, we used the Back-propagation Neural Networks, which is the most popular and the well-known neural type $[19,20]$. Usually the ANNs architecture consists of three layers, the input layer, hidden layer and the output layer. The input layer receives the input from the outside world, where it has a number of neuron equal to the number of model input. The next layer is called the hidden layer. This layer receives the input from the direct prior layers. The last layer is the output layer, used to produce the output as its name. Neurons in the same layer are not connected to each other but the neurons in each layer were fully connected to all neurons in the next layer. The neurons weights were adjusted using the activation (i.e. sigmoid) function argument. This activation function is assumed to be nonlinear [21]. Let $n_{1}(p), n_{2}(p)$, $\cdots, n_{n}(p)$ be the network inputs, and let $m_{d, 1}(p), m_{d, 2}(p), \cdots$, $m_{d, n}(p)$ be the estimated output. The back propagation neural network function can be also explained as [13].

1) The output from the hidden layer is calculated using Equation (1).

$$
m_{j}(p)=\operatorname{sigmoid}\left[\sum_{i=1}^{n} n_{i}(p) \times w_{i j}(p)-\theta_{j}\right]
$$

Where $w_{i j}$ are the weights between the input layer and the hidden layer and between the hidden layer and the output layer, is a threshold value.

The sigmoid function is presented in Equation (2).

$$
m_{j}(p)=\frac{1}{1+\mathrm{e}^{-n_{j}(p)}}
$$

The output from the output layer is calculated using Equation (3).

$$
m_{j}(p)=\operatorname{sigmoid}\left[\sum_{i=1}^{n} n_{i}(p) \times w_{i j}(p)-\theta_{j}\right]
$$

The Error Gradient from the output layer is calculated from Equation (4).

$$
\delta_{k}(p)=m_{k}(p) \times\left[1-m_{k}(p)\right] \times \mathrm{e}_{k}(p)
$$

where $\mathrm{e}_{k}(p)$ is the error at the output layer

$$
\mathrm{e}_{k}(p)=m_{d, k}(p)-m_{k}(p)
$$

2) The ANNs weight can be computed as given in Equation (6).

$$
\Delta w_{j k}(p)=\alpha \times m_{j}(p)+\delta_{k}(p)
$$

To readjust the weights of the ANNs we use Equation (7).

$$
w_{j k}(p+1)=w_{j k}(p)+\Delta w_{j k}(p)
$$

The gradient error in the hidden layer is calculated from Equation (8).

$$
\delta_{k}(p)=m_{j}(p) \times\left[1-m_{j}(p)\right] \times \sum_{k=1}^{i} \delta_{k}(p) \times w_{j k}(p)
$$

3) Calculating the weights again from Equation (9).

$$
\Delta w_{i j}(p)=\alpha \times n_{i}(p)+\delta_{j}(p)
$$

4) A gain we readjust the weights by Equation (10).

$$
w_{i j}(p+1)=w_{i j}(p)+\Delta w_{i j}(p)
$$

The back propagation algorithm can be simply explained and shown from the flow chart in Figure 1.

\section{Neural Network Model for Carbon Emission Estimation Problem}

The neural network structure that used for the carbon estimation is a multi-layer feed forward network. As explained before the network consists of an input layer, one hidden layer, and an output layer. The input layer consists of four inputs data the global oil, natural gas, coal, and primary energy consumption. The hidden layer function is a nonlinear and consists of 5 neurons. The hidden units

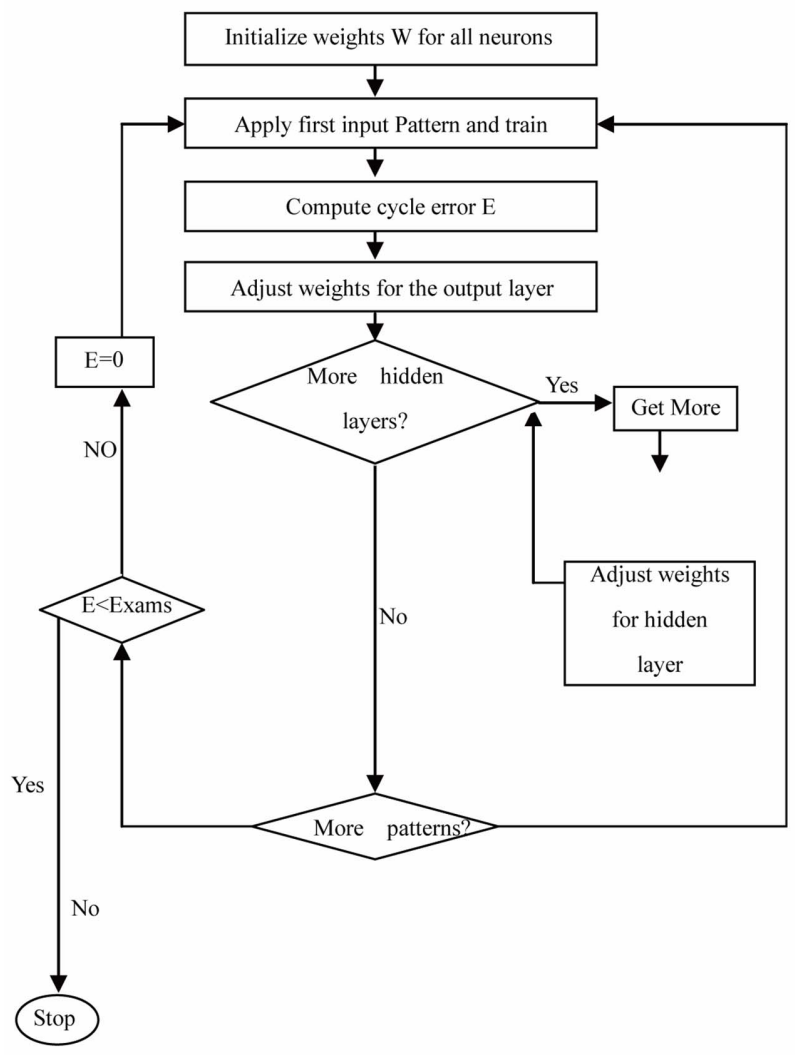

Figure 1. Back propagation flow network diagram. 
are fully mapped and connected to both the input and output. The activation function of the hidden units provides the network nonlinearity. The neurons optimal number of the hidden layer was selected by several trials. The network was trained using the Back Propagation (BP) algorithm. The number of neurons in hidden layer is selected to be 5 . The output layer consists of one output neuron producing the corresponding carbon emission estimation. The output layer node has a linear activation function. The ANN developed models is shown in Figure 2.

\section{Proposed Model Structure and Evaluation Criterion}

In our case we used four inputs to estimate the $\mathrm{CO}_{2}$ emission. The inputs are: Oil ( $\mathrm{t}-1)$, Oil ( $\mathrm{t}-2), \mathrm{NG}(\mathrm{t}-1)$, NG (t-2), Coal (t-1), Coal (t-2), PE (t-1), PE (t-2) and the output is the $\mathrm{CO}_{2}(\mathrm{t})$, where the inputs are measured in (Mote) and the output is measured in (Mt). The proposed network architecture was able to produce a very excellent estimation results in both training and testing cases with a very small number of differences. The neural network has 5 neurons in the hidden layers and one neuron in the output layer. The values of global oil, natural gas, coal, and primary energy consumption were obtained from $[5,22]$. The data in Table 1 were trained from the year 1982 to year 2000, and tested for the year 2003 to year 2010. In this paper we used different validation criterion to find out the percentage of error difference between the actual and estimated values as shown in Equations (11)(13).

Manhattan distance

$$
\mathrm{MD}=\left(\sum_{i=1}^{n}\left|y_{i}-\hat{y}_{i}\right|\right)
$$

Euclidian distance

$$
\mathrm{ED}=\sqrt{\left(\sum_{i=1}^{n} y_{i}-\hat{y_{i}}\right)^{2}}
$$

Mean magnitude of relative error

$$
\operatorname{MMRE}=\frac{1}{N} \sum_{i=1}^{N} \frac{\left|y_{i-} \hat{y_{i}}\right|}{y_{i}}
$$

where $y$ and $\hat{y}$ are the actual and estimated values based on the proposed model and $\mathrm{N}$ is the number of measurements used in the experiment, respectively. The neural network back propagation learning algorithm was able to perform the task properly by propagating the error each time reaching to the minimum error difference between the actual and the estimated values. In Figure 3, we show The ANN convergence curve. Figure 4 shows the actual and estimated values in both training and test- ing cases. The actual and estimated values were presented in bar forms where blue bars are the actual values and red bars are the estimated one. The different validation criteria performance evaluations are shown in Table 2.

\section{Conclusion}

In this study, we proposed an Artificial Neural Network

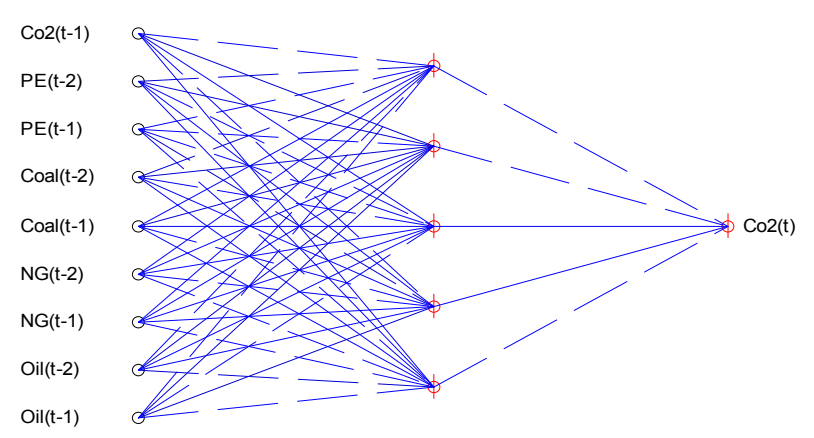

Figure 2. Developed neural network structure.

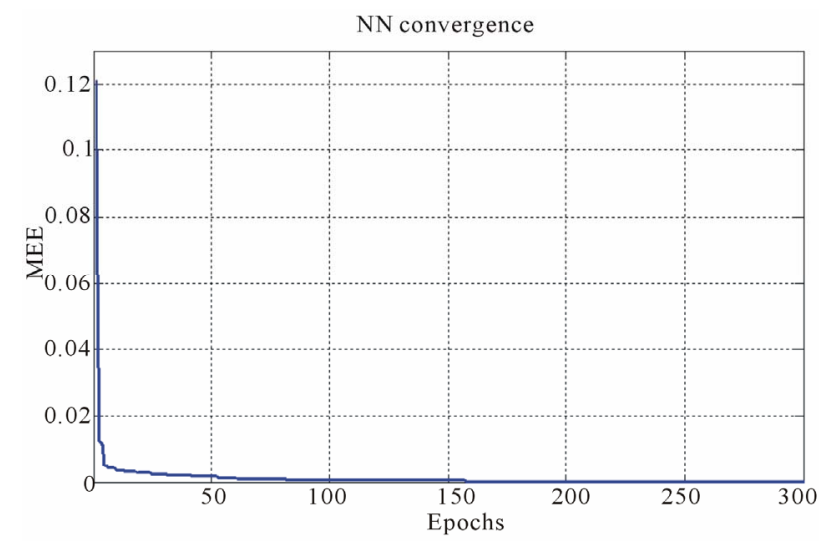

Figure 3. NNs convergence for 4 input and 1 output model.
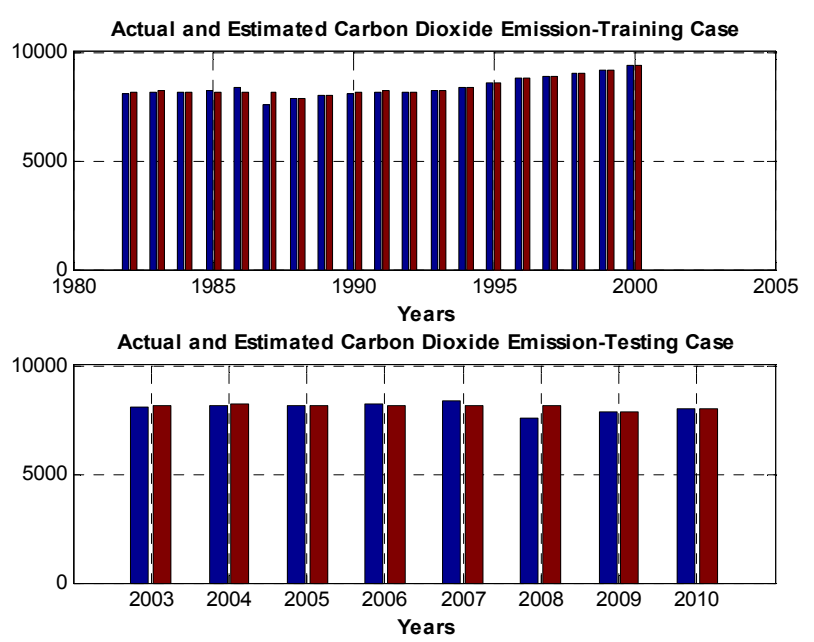

Figure 4. Actual and estimated carbon emission in both training and testing cases. 
Table 1. The data values of global oil, natural gas, coal, primary energy consumption and $\mathrm{CO}_{2}$ emission [22].

\begin{tabular}{|c|c|c|c|c|c|}
\hline Year & Oil & NG & Coal & PE & $\mathrm{CO}_{2}$ \\
\hline & Consumption & Consumption & Consumption & Consumption & Emission \\
\hline & (Mote) & (Mote) & (Mote) & (Mote) & (Mt) \\
\hline 1980 & 2972.2 & 1296.9 & 1806.4 & 6624 & 19322.4 \\
\hline 1981 & 2863 & 1309.5 & 1820.6 & 6577.5 & 19073.2 \\
\hline 1982 & 2770.7 & 1312.5 & 1846.9 & 6548.4 & 18900.7 \\
\hline 1983 & 2748.3 & 1329 & 1897.7 & 6638.2 & 19072.1 \\
\hline 1984 & 2810.1 & 1440 & 1983.2 & 6960.2 & 19861 \\
\hline 1985 & 2804.7 & 1488.3 & 2056 & 7137.5 & 20246.7 \\
\hline 1986 & 2894.1 & 1503.6 & 2089.2 & 7307.5 & 20688.3 \\
\hline 1987 & 2946.8 & 1579.6 & 2169 & 7555.7 & 21344.5 \\
\hline 1988 & 3038.8 & 1654.9 & 2231.7 & 7833.5 & 22052.2 \\
\hline 1989 & 3093 & 1729.2 & 2251.2 & 8001.7 & 22470.2 \\
\hline 1990 & 3148.6 & 1769.5 & 2220.3 & 8108.7 & 22613.2 \\
\hline 1991 & 3148.2 & 1807.5 & 2196.4 & 8156 & 22606.5 \\
\hline 1992 & 3184.8 & 1817.9 & 2174.6 & 8187.6 & 22656.7 \\
\hline 1993 & 3158 & 1853.9 & 2187.6 & 8257.5 & 22710.6 \\
\hline 1994 & 3218.7 & 1865.4 & 2201.9 & 8357.6 & 22980.3 \\
\hline 1995 & 3271.3 & 1927 & 2256.2 & 8577.9 & 23501.7 \\
\hline 1996 & 3344.9 & 2020.5 & 2292.2 & 8809.5 & 24089.8 \\
\hline 1997 & 3432.2 & 2016.8 & 2301.8 & 8911.6 & 24387.1 \\
\hline 1998 & 3455.4 & 2050.3 & 2300.2 & 8986.6 & 24530.5 \\
\hline 1999 & 3526 & 2098.4 & 2316 & 9151.4 & 24922.7 \\
\hline 2000 & 3571.6 & 2176.2 & 2399.7 & 9382.4 & 25576.9 \\
\hline 2001 & 3597.2 & 2216.6 & 2412.4 & 9465.6 & 25800.8 \\
\hline 2002 & 3632.3 & 2275.6 & 2476.7 & 9651.8 & 26301.3 \\
\hline 2003 & 3707.4 & 2353.1 & 2677.3 & 9997.8 & 27508.7 \\
\hline 2004 & 3858.7 & 2431.8 & 2858.4 & 10482 & 28875.2 \\
\hline 2005 & 3908.5 & 2511.2 & 3012.9 & 10800.9 & 29826.1 \\
\hline 2006 & 3945.3 & 2565.6 & 3164.5 & 11087.8 & 30667.6 \\
\hline 2007 & 4007.3 & 2661.3 & 3305.6 & 11398.4 & 31641.2 \\
\hline 2008 & 3996.5 & 2731.4 & 3341.7 & 11535.8 & 31915.9 \\
\hline 2009 & 3908.7 & 2661.4 & 3305.6 & 11363.2 & 31338.8 \\
\hline 2010 & 4028.1 & 2858.1 & 3555.8 & 12002.4 & 33158.4 \\
\hline
\end{tabular}

Table 2. MD, ED and MMER for ANN model training and testing data of the carbon estimation.

\begin{tabular}{cccc}
\hline Model & MD & ED & MMRE \\
\hline Training & 61.1885 & 614.2148 & 0.0078 \\
Testing & 125.6023 & 606.6500 & 0.0160 \\
\hline
\end{tabular}

model to estimate the values of the carbon dioxide emitted. The ANN was trained by the backpropagation learning algorithm. The proposed ANN model results show that ANN was capable of producing high estimation capabilities. This is clearly seen from the obtained results and the shown relationship between the actual and estimated responses. Again the ANNs proved its ability in solving the carbon estimating problem from a given set of examples. We plan to explore the use of other soft computing techniques to solve this problem such as fuzzy logic and genetic programming.

\section{REFERENCES}

[1] M. R. Lotfalipour, M. A. Falahi and M. Ashena, "Economic Growth, $\mathrm{CO}_{2}$ Emissions, and Fossil Fuels Consumption in Iran," Energy Policy, Vol. 2010, No. 35, 2010, pp. 5115-5120.

[2] H. Davoudpour and M. S. Ahadi, "The Potential for Greenhouse Gases Mitigation in Household Sector of Iran: Cases of Price Reform/Efficiency Improvement and Scenario for 2000-2010," Energy Policy, Vol. 34, No. 1, 2006, pp. 40-49.

[3] I. A. Samoilov, A. I. Nakhutin, "Esimation and Med- 
dium-Term Forecasting of Anthropogenic Carbon Dioxide and Methods Emission in Russia with Statistical Methods," Vol. 34, No. 6, 2009, pp. 348-353.

[4] W. David, "Reduction in Carbon Dioxide Emissions: Estimating the Potential Contribution from Wind Power," Renewable Energy Foundation, December 2004.

[5] M. A. Behrang,. E. Assareh, M. R. Assari and A. Ghanbarzadeh, "Using Bees Algorithm and Artificial Neural Network to Forecast World Carbon Dioxide Emission," Energy Sources, Part A: Recovery, Utilization, and Environmental Effects, Vol. 33, No. 19, 2011, pp. 1747-1759.

[6] H. T. Pao and C. M. Tsai, "Modeling and Forecasting the $\mathrm{CO}_{2}$ Emissions, Energy Consumption, and Economic Growth in Brazil," Energy, Vol. 36, No. 5, 2011, pp. 2450-2458.

[7] N. Karunanithi, W. Grenney, D. Whitley and K. Bovee, "Neural Networks for River Flow Prediction," Journal of Computing in Civil Engg, Vol. 8, No. 2, 1993, pp. 371379.

[8] P. R. Bulando and J. Salas, "Forecasting of Short-Term Rainfall Using ARMA Models," Journal of Hydrology, Vol. 144, No. 1-4, 1993, pp. 193-211.

[9] H. Hruschka, "Determining Market Response Functions by Neural Networks Modeling: A Comparison to Econometric Techniques," European Journal of Operational Research, Vol. 66, 1993, pp. 867-888.

[10] E. Y. Li, "Artificial Neural Networks and Their Business Applications," Information and Managements, Vol. 27, No. 5, 1994, pp. 303-313.

[11] K. Chakraborty, "Forecasting the Behavior of Multivariable Time Series Using Neural Networks," Neural Networks, Vol. 5, 1992, pp. 962-970.

[12] G. Swales and Y. Yoon, "Applying Artificial Neural Networks to Investment Analysis," Financial Analyst Journal, Vol. 48, No. 5, 1992, pp. 78-82.

[13] M. Negnevissky, "Artificial Intelligence: A Guide to Intelligent Systems," 2nd Edition, Addison-Wesley, Boston, 2005.

[14] E. Y. Li, “Artificial Neural Networks and Their Business
Applications," Information and Managements, Vol. 27, No. 5, 1994, pp. 303-313.

[15] A. K. Baareh, A. Sheta and K. Al Khnaifes, "Forecasting River Flow in the USA: A Comparison between AutoRegression and Neural Network Non-Parametric Models," Journal of Computer Science, Vol. 2, No. 10, 2006, pp. 775-780.

[16] M. S. Al-Batah, I. Mat, K. Z. Zamli and K. A. Azizli, "Modified Recursive Least Squares Algorithm to Train the Hybrid Multilayered Perceptron (HMLP) Network," Applied Soft Computing, Vol. 10, No. 1, 2010, pp. 236244.

[17] M. Seethe, I. V. Muralikrisha and B. L. Deekshatulu, "Artificial Neural Networks and Other Methods of Image Classifications," Journal of Theoretical and Applied Information Technology, Vol. 4, No. 11, 2005, pp. 10391053.

[18] H. J. Lu, R. Setiono and H. Lui, "Effective Data Mining Using Neural Networks," IEEE Transactions on Knowledge and Data Engineering, Vol. 8, No. 6, 1996, pp. 957961.

[19] O. Kisi, "River Flow Modeling Using Artificial Networks" Journal of Hydrologic Engineering, Vol. 9, No. 1, 2004, pp. 60-63.

[20] H. K. Cigizoglu and O. Kisi, "Flow Prediction by Three Back-Propagation Techniques Using K-Fold Partitioning of Neural Network Training Data," Nordic Hydrology, Vol. 36, No. 1, 2005, pp. 49-64.

[21] A. K. Baareh, A. Sheta and K. Al Khnaifes, "Forecasting River Flow in the USA: A Comparison between AutoRegression and Neural Network Non-Parametric Models," Proceedings of the 6th WSEAS International Conference on Simulation, Modelling and Optimization, Lisbon, 22-24 September 2006, pp. 7-12.

[22] H. Kavoosi, M. H. Saidi, M. Kavoosi and M. Bohrng, "Forecast Global Carbon Dioxide Emission by Use of Genetic Algorithm (GA)," IJCSI International Journal of Computer Science Issues, Vol. 9, No. 5, 2012, pp. 418427. 